\section{ESCASAS CIRUGÍAS CARDIOVASCULARES EN NIÑOS CON CARDIOPATÍAS CONGÉNITAS EN AREQUIPA, PERÚ}

\section{INSUFFICIENT CARDIOVASCULAR \\ SURGERIES IN CHILDREN WITH CONGENITAL HEART DEFECTS IN AREQUIPA, PERU}

Jersson Estefanero-Meza ${ }^{1, a}$, Daniel Pinto-Torres ${ }^{1, a}$,
Gaby Mamani-Huaman', ${ }^{2, b}$, Oscar Moreno-Loaiza ${ }^{3, b}$

Sr. Editor. En el Perú, se ha estimado que para el periodo 2006-2010 el número total de cardiopatías congénitas osciló entre 3888 y 3925 casos (1). Existen, además, reportes de mayor incidencia de cardiopatías congénitas en poblaciones que viven a grandes alturas. Esta situación se agrava en regiones donde existe una pobre oferta de servicios de salud especializados ${ }^{(2,3)}$. Siendo considerado como un problema de salud pública debido a la morbi-mortalidad asociada a estas enfermedades.

Se desconoce la incidencia de cardiopatías congénitas atendidas en Arequipa, ciudad en la que se atienden pacientes no solo del departamento, sino también aquellos que son referidos de ciudades como Cusco y Puno, ubicadas a más de $3000 \mathrm{~m}$ de altitud. Esto incrementa el número de atenciones que se brindan por cardiopatías congénitas en los hospitales de esta ciudad.

En los hospitales del Ministerio de Salud (MINSA) de Arequipa, hemos observado que se realizan escasas cirugías cardiovasculares pediátricas, y que dicho problema es solucionado parcialmente por el apoyo de misiones extranjeras. El objetivo de esta comunicación es evidenciar la incidencia de cardiopatías congénitas y las cirugías cardiovasculares realizadas en el Hospital Regional Honorio Delgado Espinoza (HRHDE) del MINSA en Arequipa, Perú.

\footnotetext{
Centro de Investigación y Estudios Médicos, Facultad de Medicina, Universidad Católica de Santa María. Arequipa, Perú.

2 Puesto de Salud de Corani. Puno, Perú.

3 Centro de Salud Hunter. Arequipa, Perú.

Estudiante de Medicina; ${ }^{b}$ médico cirujano.

Recibido: 26-08-2013 Aprobado: 04-09-13
}

Citar como: Estefanero-Meza J, Pinto-Torres D, Mamani-Huaman G, Moreno-Loaiza O. Escasas cirugías cardiovasculares en niños con cardiopatías congénitas en Arequipa, Perú [carta]. Rev Peru Med Exp Salud Publica. 2013;30(4):716-7.
Revisamos las historias clínicas de los recién nacidos con cardiopatías congénitas durante los años 2010-2012; así mismo, buscamos el total de casos de cardiopatías congénitas atendidas y las cirugías cardiovasculares realizadas en pacientes pediátricos.

En el periodo 2010-2012 se atendieron 210 pacientes pediátricos con diagnósticos de cardiopatías la edad de los niños oscila entre 1,7 años $\pm 3,6$; el 22,8\% (48) de estos pacientes fallecieron. La incidencia de cardiopatías congénitas fue de 2,3 casos por mil recién nacidos vivos, siendo los diagnósticos los que se expresan en la Tabla 1.

Tabla 1. Cardiopatías congénitas en recién nacidos del Hospital Regional Honorio Delgado Espinoza, Arequipa, Perú 2010-2012

\begin{tabular}{lcc}
\hline \multicolumn{1}{c}{ Cardiopatía congénita } & N & (\%) \\
\hline Cardiopatías acianóticas* $^{*}$ & 29 & $(67,4)$ \\
Persistencia del conducto arterioso & 8 & $(18,6)$ \\
Comunicación interventricular & 7 & $(16,3)$ \\
Comunicación interauricular & 5 & $(11,6)$ \\
Cardiopatías cianóticas* $^{*}$ & 5 & $(11,6)$ \\
Tetralogía de Fallot & 1 & $(2,3)$ \\
Anomalía de Ebstein & 1 & $(2,3)$ \\
\hline
\end{tabular}

* Diagnósticos no especificados

Se realizaron 25 cirugías cardiovasculares pediátricas, lo que corresponde al $20,4 \%$ de pacientes con cardiopatías congénitas, el $88 \%$ (22) de las cirugías fueron realizadas por el equipo médico de "CardioStart International", el resto de cirugías fueron realizadas por médicos del HRHDE. Ninguno de los pacientes operados falleció.

Los estudios sobre las frecuencias de cardiopatías congénitas colocan en primeros lugares de incidencia a la comunicación interventricular (CIV) y la comunicación interauricular $(\mathrm{CIA})^{(1,3)}$. En el caso del HRHDE la mayoría de las cardiopatías no fueron especificadas, sea por falta de recursos por parte de los pacientes, y el hospital.

Las misiones extranjeras han demostrado ayudar a mejorar las cirugías cardiovasculares en países en desarrollo como Guatemala, en donde la experiencia con especialistas extranjeros ayudó a disminuir la mortalidad posoperatoria de pacientes con cardiopatías congénitas ${ }^{(4)}$. Sin embargo, en Arequipa observamos que el principal problema es la pobre cantidad de cirugías cardiovasculares realizadas; siendo en ese aspecto dependientes casi completamente de misiones extranjeras.

La escasa realización de cirugías cardiovasculares en provincias podría deberse a la falta de especialistas, así como a la capacitación de estos en el manejo de 
pacientes pediátricos. Por otro lado, la falta de material quirúrgico y protésico podría ser otro limitante, que se ve resuelto cuando las misiones extranjeras traen consigo dicho material. Esto constituye una situación de inequidad en cuanto al acceso a servicios de salud por parte de la población no asegurada y la que no cuenta con recursos para acceder a sistemas privados de salud. Para solucionar este problema se ha visto que el referir pacientes a otros países resulta costoso, y no crea experiencia institucional ni recursos humanos para resolver el problema local ${ }^{(5)}$.

Traer o esperar el apoyo de cirujanos extranjeros, como en Arequipa, es una solución parcial que queda desaprovechada si es que no se utiliza esta experiencia para capacitar a más personal y generar especialistas. Así mismo, estos procedimientos requieren de un manejo multidisciplinario para la prevención y tratamiento de las complicaciones posquirúrgicas, que podría no ser resuelto a nivel local.

La brecha entre oferta y demanda de especialidades médicas en el Perú es alta, sobre todo fuera de la capital del país ${ }^{(2)}$. El número de especialistas de cirugía cardiovascular a nivel nacional ocupa el penúltimo lugar entre las especialidades quirúrgicas ${ }^{(2)}$. Este problema requiere el incremento de campos clínicos y quirúrgicos para la formación de estos especialistas, así como una mayor oferta de residencias médicas que incluyan, además, especialidades como cardiología pediátrica, y medicina intensiva pediátrica ${ }^{(5)}$.

Los recursos económicos y materiales también son parte de este problema, para cuya solución se necesitan cambios en los aspectos administrativos y del Seguro Integral de Salud, a fin de garantizar una atención oportuna y que reduzca las complicaciones de las cardiopatías congénitas en los pacientes pediátricos.

Agradecimientos: al Dr. Manuel García Vela, médico pediatra asistente del Departamento de Pediatría del Hospital Regional Honorio Delgado Espinoza, Arequipa, Perú, quien permitió la recolección de los datos.

Fuentes de financiamiento: autofinanciado.

Conflicto de intereses: los autores declaran no tener conflictos de interés.

\section{REFERENCIAS BIBLIOGRÁFICAS}

1. Olórtegui A, Adrianzén M. Incidencia estimada de las cardiopatías congénitas en niños menores de 1 año en el Perú. An Fac Med. 2007;68(2):113-24.
2. Zevallos L, Pastor R, Moscoso B. Oferta y demanda de médicos especialistas en los establecimientos de salud del Ministerio de Salud: brechas a nivel nacional, por regiones y tipo de especialidad. Rev Peru Med Exp Salud Publica. 2011;28(2):177-85.

3. Chen $\mathrm{QH}$, Wang XQ, Qi SG. Cross-sectional study of congenital heart disease among Tibetan children aged from 4 to 18 years at different altitudes in Qinghai Province. Chin Med J (Engl). 2008;121(24):2469-72.

4. Larrazabal LA, Jenkins KJ, Gauvreau K, Vida VL, Benavidez OJ, Gaitán GA, et al. Improvement in congenital heart surgery in a developing country: the Guatemalan experience. Circulation.. 2007;116(17):1882-7.

5. Stolf NA. Congenital heart surgery in a developing country: a few men for a great challenge. Circulation. 2007;116(17):1874-5.

Correspondencia: Jersson Estefanero Meza

Dirección: Calle Espinar 1200, Miraflores, Arequipa, Perú.

Teléfono: (511) 957465206

Correo electrónico:ccvjaem@gmail.com

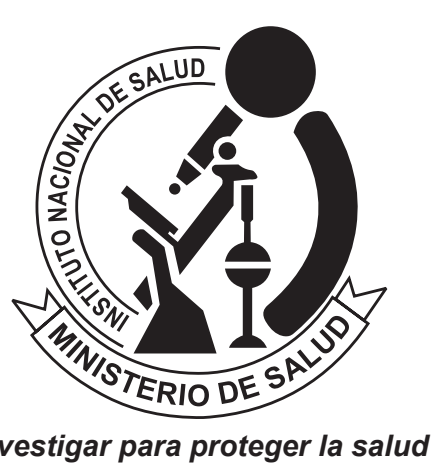

REVISTA PERUANA DE MEDICINA EXPERIMENTAL Y SALUD PÚBLICA CUMPLIENDO SUS METAS Y PROYECTÁNDOSE AL FUTURO

\section{Visite los contenidos de la revista en: www.ins.gob.pe/rpmesp}

